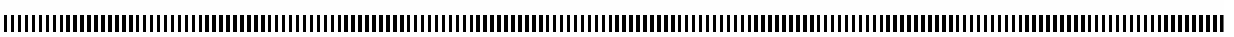

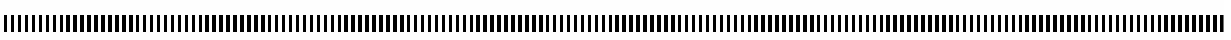

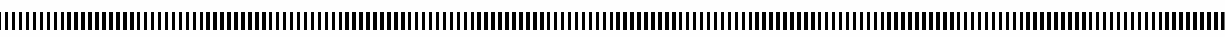

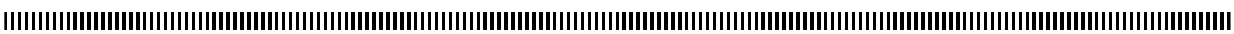

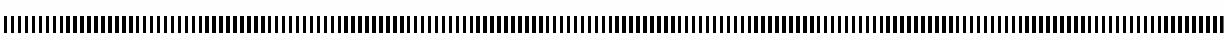

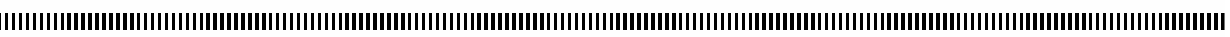

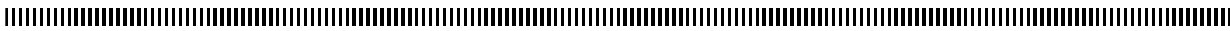

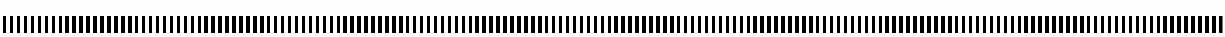

\title{
Simulation du remplissage des moules par la méthode des éléments Finis / volume contrôle dans les procédés RTM
}

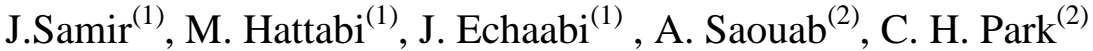 \\ 1. Équipe de Recherche Appliqué sur les polymères, Département de Génie Mécanique, \\ ENSEM, Université Hassan II Aïn Chok, BP : 8118, Oasis, Casablanca, Maroc. \\ 2. Laboratoire de Mécanique, Physique et Géosciences, Université du Havre \\ 25 rue Philippe Lebon, BP 540, 76058, Le Havre, France
}

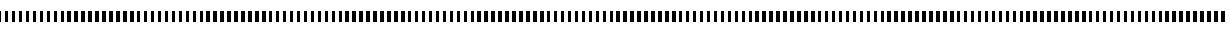

RÉSUMÉ. La présente étude porte sur la simulation du remplissage isotherme des moules en procédé RTM, en adoptant la méthode CV/FEM et VOF, avec prise en compte de la présence d'obstacles couplée à l'effet de la variation de l'épaisseur et des couches du renfort. Les codes développés ont pour objectif d'optimiser le positionnement des points d'injection, des évents et déterminer les pressions d'injection optimales pour minimiser les temps de cycle. Ces codes utilisent un mailleur propre à l'équipe permettant de discrétiser le domaine en éléments triangulaires non structurés avec possibilité de raffinement local et d'inclusion d'inserts.

ABSTRACT. In the course of this study, the simulation of the resin flow in the RTM process is developed by the control volume finite element method (CVFEM) coupled with the equation of the free surface location. This location is made by means of the so called "Volume of Fluid" methods or VOF. Thus, the position of the flow front, the time-lapse and the rate of the non saturated zone are calculated at every step. Our results will be compared with the experimental and analytical models in the literature. On the whole, our study is concerned with the simulation of the thermally insulated filling of moulds in RTM process while adopting the CVFEM and VOF method, taking into account the presence of obstacles, coupled with the thickness variation effect and the reinforcement coats.

MOTS-CLÉS : RTM, VOF, CV/FEM, simulation, écoulement.

KEYWORDS: RTM, VOF, CV/FEM, simulation, flow.

|

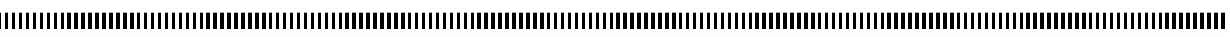

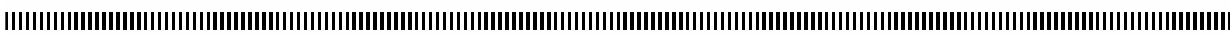

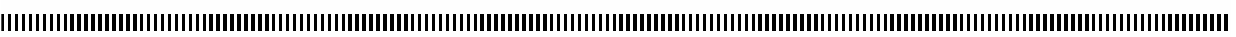

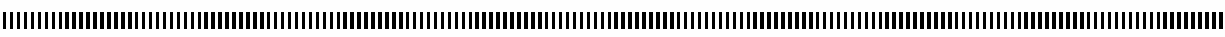

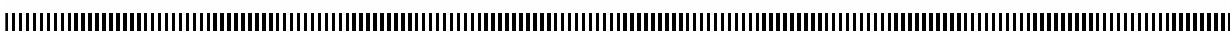

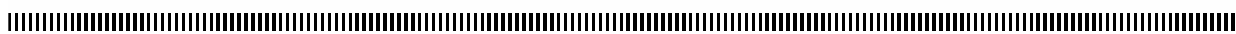

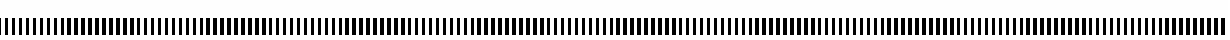

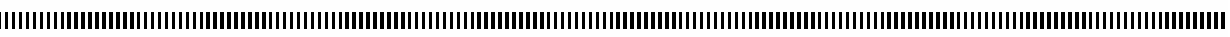
|

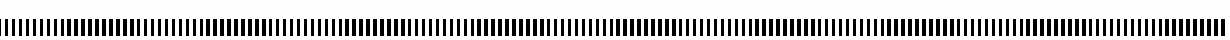




\section{Introduction}

Aujourd'hui, le RTM reste un des procédés de fabrication des composites les plus avantageux, par son coût de réalisation, sa productivité élevée et ses possibilités de mise en œuvre des pièces à forme complexe et caractéristiques mécaniques remarquables. Son principe général consiste à injecter dans un moule fermé, une résine thermodurcissable sur un renfort fibreux préalablement disposé. Il comporte deux sous familles : les procédés RTM (Resin Transfert Molding) et ses dérivées et les procédés d'infusion et ses dérivées. Dans le premier cas la résine s'imprègne dans le renfort par un mode de transfert et dans le second par infusion. Les applications de ce procédé sont de plus en plus pointues et permettent de réaliser des pièces de bonne qualité dans diverses applications. La présente étude concerne la modélisation numérique des procédés LCM. Quelques travaux ont proposé des méthodes basées sur les systèmes des coordonnées curvilignes adaptés à une frontière [1]. Cependant, les limites de cette approche se sentent lors des divisions ou fusions des fronts d'écoulement [2,3]. Actuellement, les méthodes de modélisation développées sont basées sur une approche de type éléments finis couplé à des volumes de contrôle. Ce type d'approche a été proposé pour la première fois par Wang et autres, il a été adopté dans le cas du moulage par injection des coquilles minces [4]. Fracchis était le premier à proposer la méthode de FE/CV pour simuler le processus RTM [5] et d'autres chercheurs ont également suivi cette approche [6-9]. A travers cette étude, nous proposons un mailleur 2 et $2.5 \mathrm{D}$ et une méthode numérique pour la simulation du remplissage des moules dans le procédé RTM avec prise en charge de la variation d'épaisseur du renfort. La méthode numérique adoptée est basée sur une discrétisation type éléments finis couplé au volume de contrôle (CV/MEF), la perméabilité et la porosité sont des propriétés relatives à l'élément du domaine discrétisé. Des exemples numériques sont fournis pour valider et évaluer l'applicabilité du code développé.

\section{Formalisme mathématique}

\subsection{Equations de Continuité et la loi de Darcy}

En RTM, au cours du remplissage du moule, l'écoulement (résine) traverse un lit de fibre. Le processus d'injection peut être alors étudié, soit à l'échelle microscopique ou à l'échelle macroscopique. Sachant que lors de l'approche microscopique, le processus d'injection est traité au niveau du champ local de l'écoulement entre les faisceaux de fibres (étude complexe), l'approche macroscopique est devenue, en conséquence, l'outil commun pour la simulation du procédé RTM. Au cours de cette méthodologie, le processus d'injection dans le moule est traité dans le cadre des écoulements des fluides à travers les milieux poreux. 
L'équation de la continuité pour le fluide peut être écrite comme :

$$
\frac{\partial v_{i}}{\partial x_{i}}=0
$$

Avec $v_{i}$ le vecteur de vitesse.

Comme l'écoulement du fluide passe à travers les pores du renfort, le champ de vitesse de la résine peut être décrit comme suit :

$$
v_{i}=\frac{q_{i}}{\phi}
$$

avec $q_{i}$ le vecteur de vitesse superficiel et $\phi$ la porosité du solide.

En supposant que le renfort est un milieu poreux et que l'écoulement est en état quasistable, l'équation de la vitesse peut être remplacée par la loi de Darcy:

$$
q_{i}=-\frac{K_{i j}}{\mu} \frac{\partial P}{\partial x_{j}}
$$

Avec $\mu$ est la viscosité fluide, $K_{i j}$ le tenseur de la perméabilité du renfort, et $P$ est la pression fluide.

Notons que la résine est incompressible. La substitution du (3) dans (1) donne l'équation différentielle qui gouverne l'écoulement:

$$
\frac{\partial}{\partial x_{i}}\left(\frac{K_{i j}}{\mu} \frac{\partial P}{\partial x_{j}}\right)=0
$$

Cette équation aux dérivés partiels du second degré peut être résolue quand les conditions aux limites sont définies.

Aux points d'injection du moule deux conditions peuvent être définis, les deux sont en fonction de la pression:

Soit la pression d'injection :

$$
P_{\text {inlet }}=P_{\text {inlet }}(t)
$$

Ou en débit d'injection:

$$
Q_{n}(t)=n_{i} \frac{K_{i j}}{\mu} \frac{\partial P}{\partial x_{j}}
$$

avec $Q_{n}$ le débit volumétriques et $n_{i}$ le vecteur normal aux points d'injection. 
Les conditions aux limites sur le front sont supposées nulles:

$$
P_{\text {front }}=0
$$

Puisque la résine ne peut pas franchir les bords du moule, la dernière condition aux limites nécessaires pour résoudre l'équation (4) est que la normale de vitesse aux bords du moule est nulle :

$$
\bar{v} \cdot \bar{n}=0
$$

avec $n$ est la normale au bord du moule.

\subsection{Discrétisation du domaine par MEF/VC-VOF}

Lors de l'adoption de la méthode MEF/VC la position du fluide dans le volume de contrôle n'est pas connue, ainsi, la forme exacte du front d'écoulement ne peut être définie. En conséquence, La qualité du front d'écoulement numérique est liée directement à la densité et la qualité du maillage.

Concernant la discrétisation du domaine de calcul, nous avons développé un mailleur permettant de :

$\checkmark$ Discrétiser le domaine en éléments triangulaires non structurés,

$\checkmark$ Inclure des inserts à différentes formes,

$\checkmark$ Raffiner le maillage des zones complexes à étudier.

En plus, le mailleur comporte une fonction de relaxation de la grille afin d'éviter la création des noeuds entourée de plus de 7 et de moins de 5 éléments (figure 1).
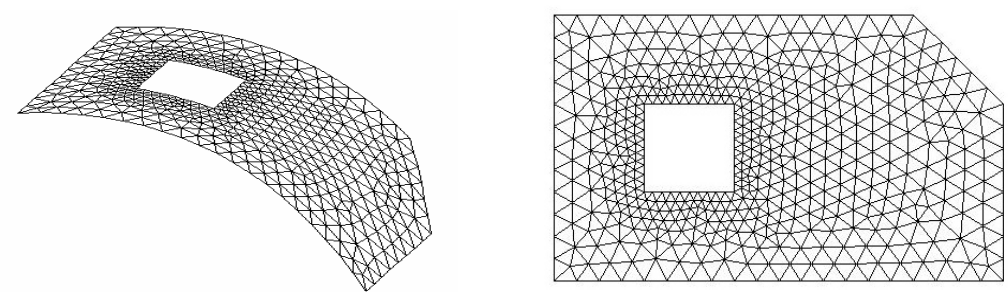

Figure 1 : Discrétisation du domaine de calcul

Dans cette étude, nous avons adopté la méthode des éléments finis couplé au volume de contrôle (FEM/CV). Le domaine de calcul est discrétisé en un nombre finis d'éléments triangulaires. En connectant les centroides des éléments et les milieux des bords des 
éléments, le domaine de calcul peut être divisé une fois de plus en un nombre de volumes de contrôle polygonaux, comme présente la figure $2 \mathrm{a}$. Les bords de chaque élément du volume du contrôle constituent le contrôle surface. Chaque volume de contrôle sera classifié selon la fraction de la résine $f$ (figure $2 \mathrm{~b}$ ).

$\checkmark f=1 \quad$ si le volume est complètement rempli ;

$\checkmark f=0 \quad$ si le volume est sec ;

$\checkmark \quad 0<f<1 \quad$ si le volume est partiellement rempli (région du front d'écoulement).
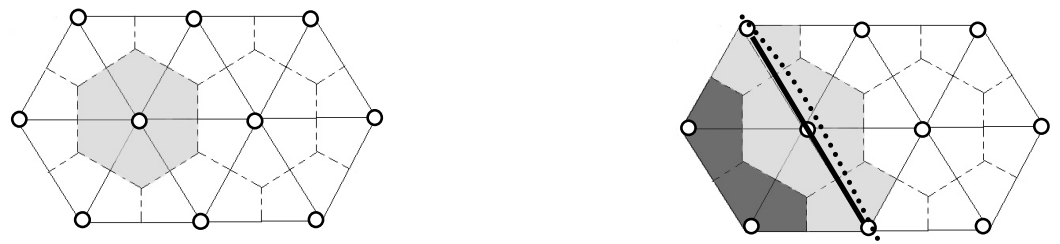

$\begin{array}{lll}\text { Nœud } & \text { Surface Contrôle } \\ \text { Bords éléments } & \text { Volume Contrôle }\end{array}$

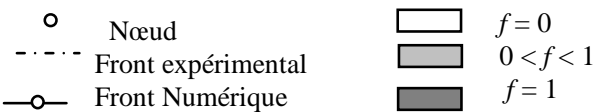

Fig. 2.a : Discrétisation en CVFEM

Fig. 2.b : Front d'écoulement en maillage fixe

Pour chaque volume contrôle, à partir de l'intégration de l'équation 2 on obtient la formule suivante :

$$
\int_{c v} \frac{\partial}{\partial x_{i}}\left(\frac{K_{i j}}{\mu} \frac{\partial P}{\partial x_{j}}\right) d \Omega=\int_{c s} \frac{K_{i j}}{\mu} \frac{\partial P}{\partial x_{i}} \vec{n} d s=0
$$

Avec $S$ la surface contrôle, $\Omega$ le volume contrôle et $\vec{n}$ le vecteur normal de la surface contrôle. Les symboles $C_{V}$ et $C_{S}$ décrivent le domaine volume contrôle et surface contrôle respectivement.

Il est à préciser qu'à chaque itération, la matrice de calcul ne contient que les éléments dont au moins un nœud est à taux de remplissage unité $f=1$. Cette approche impose une rigueur lors du développement du code, néanmoins, le temps de traitement du problème devient nettement amélioré.

\section{Simulation Numérique}

\subsection{Champ de pression}

Lors de la résolution du champ pression l'approche Galerkin a été adoptée pour décrire la distribution du champ de pression. 
L'équation gouvernant l'écoulement de la résine est :

$$
\frac{\partial}{\partial x_{i}}\left(\frac{K_{i j}}{\mu} \frac{\partial P}{\partial x_{j}}\right)=0
$$

Selon l'approche décrite par Reddy [10] la formulation éléments finis de l'équation (4) est :

$$
\left[K_{i j}^{e}\right]\left\{P_{j}^{e}\right\}=\left\{F_{i}^{e}\right\}
$$

avec

$$
\begin{gathered}
K_{i j}^{e}=\int_{\Omega_{e}} \frac{K_{\alpha \beta}}{\mu} \frac{\partial \psi_{i}}{\partial x_{\alpha}} \frac{\partial \psi_{j}}{\partial x_{\beta}} d \Omega \\
F_{i}^{e}=\int_{\Omega_{e}} f \Psi_{i} d \Omega+\int_{\Gamma_{e}} Q_{n} \Psi_{i} d \Gamma
\end{gathered}
$$

$\Omega_{e}$ est le domaine d'un élément, $\Gamma_{e}$ est la surface d'un élément, $P_{j}^{e}$ est la pression de chaque noeud, f est le terme source, $Q_{n}$ est le flux spécifique du fluide à travers la face de l'élément, et $\Psi_{i}$ est une fonction d'interpolation linéaire.

\subsection{Champ Vitesse}

Ayant utilisé l'interpolation linéaire, dans laquelle chaque élément peut être approché par des coefficients [11] et les composantes de la vitesse dans le repère principal sont calculées par :

$$
u_{i}=-\frac{k_{i i}}{\mu} \frac{\partial P}{\partial x_{i}}
$$

\subsection{Calcul des paramètres de remplissage}

La méthode de volume de contrôle permet de suivre le front d'écoulement en déterminant les volumes de contrôle partiellement remplis et en les reliant pour former le front d'écoulement. Ainsi au cours de la méthode EF/VC le front d'écoulement numérique est créé à partir des nœuds dont les volumes de contrôle sont partiellement remplis.

\section{Calcul du débit}

On suppose que la vitesse du fluide est constante à l'intérieur de l'élément. L'écoulement dans chaque volume de contrôle nodal est défini par : 


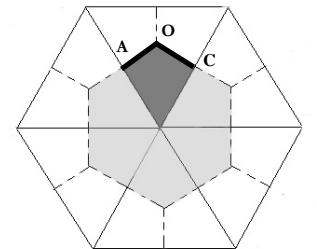

$$
Q_{e n}=\bar{v}_{e} \bar{a}_{e n}=-\int_{o}^{A} \frac{K_{i j}}{\mu} \frac{\partial P}{\partial x_{j}} \cdot \vec{n} d s
$$

Figure 3 : Calcul de la vitesse de remplissage CV/FEM

Avec $Q_{e n}$ le débit volumétrique relatif au volume de contrôle (n) de l'élément (e), $\bar{v}_{e}$ la vitesse du fluide dans l'élément, et $\bar{a}_{e n}$ le vecteur normal de l'aire du volume-secondaire lié au segment $A O$.

\section{Calcul du taux de remplissage}

Après que les débits dans chaque volume de contrôle aient été calculés les taux de remplissage peuvent être mis à jour. Pour chaque itération, à partir du taux de remplissage de l'étape précédente, du débit calculé, et du volume de chaque cv, le nouveau taux de remplissage peut être calculé selon l'équation :

$$
f_{n}^{i+1}=f_{n}^{i}+\frac{\Delta t \sum_{e} Q_{e n}}{V_{n}}
$$

Avec $f$ le taux de remplissage, $\Delta t$ le temps de l'itération et $V_{n}$ le volume du volume de contrôle.

\section{Calcul du temps de l'itération}

Le temps de l''itération suivante doit être calculé avant la mise à jour des taux de remplissage. Par définition il est le temps nécessaire pour que le fluide remplisse juste un volume de contrôle. Si une plus grande durée était choisie, le front d'écoulement déborderait du volume de contrôle et une perte de masse dans le système résulterait. Le temps de remplissage du volume de contrôle partiellement rempli $\mathrm{n}$ est calculée par la relation suivante

$$
\Delta t_{n}=\frac{\left(1-f_{n}\right) V_{n}}{\sum_{e} Q_{e n}}
$$

Une fois que $\Delta t_{n}$ a été calculé pour tous les volumes de contrôle partiellement remplis, le plus petit $\Delta t$ est choisi comme le temps de la prochaine itération. 


\section{Résultats et discussions}

Dans les exemples de validation présentés, les moules utilisés sont à cavité rectangulaire de dimensions respectives $(1000 \times 200) \mathrm{mm}^{2}$ et $(400 \times 400) \mathrm{mm}^{2}$. Le premier, concerne la validation unidirectionnelle de nos résultats numériques alors que le deuxième est exploité en bidirectionnel. Le fluide utilisé est de viscosité $\mu=0,109$ Pas injecté sous la pression $P_{i n j}=2 \times 10^{5} \mathrm{~Pa}$ à travers un renfort isotrope de perméabilité $K=2,65 \times 10^{-10} \mathrm{~m}^{2}$ et de porosité $\Phi=0,696$.

Dans ces conditions, la cinétique du front en bidimensionnel (position du front $x_{f}$ au cours du temps) peut être obtenue analytiquement par l'équation (18). La comparaison des fronts analytique, expérimental et numérique est présentée en Figure 4 :

$$
x_{f}(t)=\sqrt{\left(\frac{2 K P_{i n j}}{\phi \mu} t\right)}
$$

\subsection{Validation Unidirectionnelle :}

Le moule utilisé est à cavité rectangulaire de dimensions $(1000 \times 200) \mathrm{mm}^{2}$, l'épaisseur est uniforme de $4 \mathrm{~mm}$.
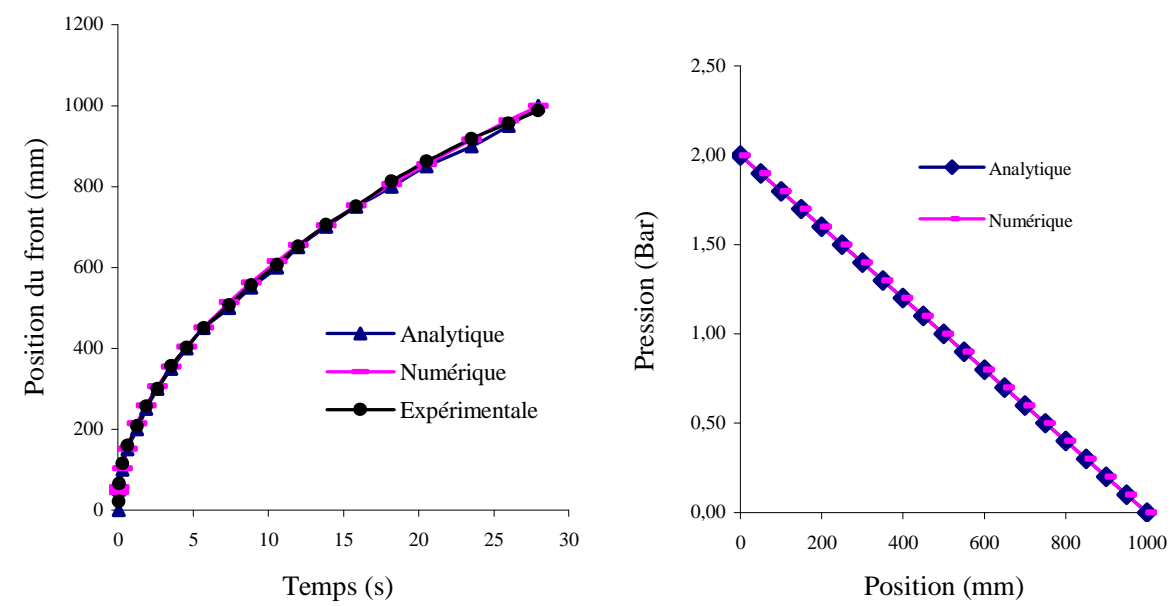

Figure 4 : Validation Unidirectionnel 


\subsection{Validation Bidirectionnelle}

Le moule utilisé est à cavité rectangulaire de dimensions $400 \mathrm{X} 400 \mathrm{~mm}^{2}$, l'épaisseur est uniforme de $4 \mathrm{~mm}$.

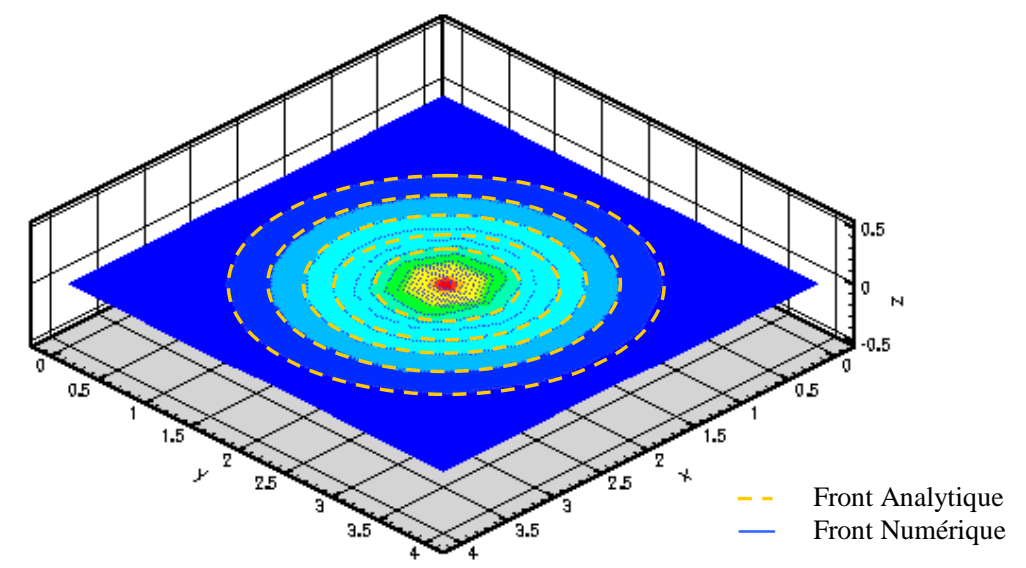

Figure 5 : Evolution du front numérique et analytique pour écoulement Bidirectionnel avec $K_{l}=K_{2}$

\subsection{Effet de variation d'épaisseur sur l'avancement du front :}

Le procédé de fabrication des matériaux composites RTM (Resin Transfer Molding) est utilisé pour produire des pièces de géométrie complexe. Dans l'industrie des composites, les plaques utilisées sont souvent constituées de renforts dont le nombre de plis (épaisseur) et les séquences d'empilements sont variables. Une simulation correcte du processus impose alors la prise en compte de l'influence de ces paramètres. La prédiction des effets de ces variations géométriques sur la transmission des ultrasons a été modélisée par l'équipe du S.Lonne suivant un formalisme dérivé de la méthode de Thomson-Haskell [12].

La variation de l'épaisseur du renfort provoque lors de la fermeture du moule, sous l'effet de la compressibilité ou de la relaxation des plaques du moule, une variation du volume et de la distribution des pores à travers le tissu, ces variations influencent directement sur deux paramètres essentiels à la simulation des écoulements en milieux poreux qui sont la perméabilité et la porosité.

La porosité d'un milieu caractérise l'importance de vides (pores) qu'il contient. Elle représente donc la fraction volumique de vide c.à.d le rapport entre le volume de l'espace poreux et le volume total du milieu poreux (équation 19) : 


$$
\phi=\frac{V_{\text {pores }}}{V_{\text {total }}}
$$

Alors que la perméabilité est définie comme étant la propriété d'un milieu poreux qui caractérise la facilité avec laquelle un fluide peut être injecté pour traverser un matériau (renfort) grâce à un gradient de pression appliqué (force motrice) entre le point d'injection et la sortie d'un moule. La perméabilité est un paramètre clé permettant d'englober l'ensemble des effets dus à l'architecture géométrique du milieu poreux constitué par le renfort. Il est difficile de prévoir analytiquement la perméabilité d'un renfort avec une architecture compliquée. Nous proposons plusieurs modèles qui donnent la perméabilité en fonction du taux de fibres. Le plus célèbre d'entre eux étant le modèle de KozenyCarman.

$$
K=\frac{1}{k_{0}\left(L_{e} / L\right)^{2}} \frac{r_{f}{ }^{2}}{4} \frac{\phi^{3}}{(1-\phi)^{2}}
$$

Avec $\emptyset$ taux de fibre $r_{f}$ rayon de la fibre et $k_{0}$ étant la constante de Kozeny-Carman, qui englobe un facteur de forme géométrique représentant différentes géométries de particules imprégnées par le fluide et $k_{1}=\left(\mathrm{L}_{\mathrm{e}} / \mathrm{L}\right)^{2}$ le facteur de la tortuosité.

Les études de Chen et al. [13] ont montré que la compressibilité initiale des renforts est essentiellement due à la compressibilité des pores, cette compressibilité « ou relaxation » influence directement sur le volume global et la distribution des pores. Ainsi, la variation de l'épaisseur du renfort provoque sous l'effet de la compressibilité ou de la relaxation une variation du volume et de la distribution des pores à travers le tissu. Les travaux de Buntain, et Bickerton [14] se sont axés sur l'effet de compressibilité sur la perméabilité, les résultats montraient de façon clair que la perméabilité (propriété indispensable à maîtriser pour une simulation correcte du front d'écoulement et de la distribution de la pression) est étroitement liée à la fraction de volume des pores.

Dans notre étude, la variation du nombre de plis et des séquences d'empilements sera modélisée par une variation de la perméabilité et de la porosité. Ces paramètres sont définis à l'échelon de l'élément et non comme propriété intrinsèque du domaine global discrétisé.

$\checkmark \quad$ Case 1D :

Dans le paragraphe suivant, est étudié le cas d'une injection en RTM unidirectionnelle multi-épaisseur. Le renfort utilisé est composé de deux parties du même tissu mais à nombre de pli différent (figure.6). Sachant que la cavité du moule est uniforme, la variation d'épaisseur entre les deux parties influence directement sur la compressibilité/relaxation du renfort pour chaque zone lors de la fermeture du moule. La compressibilité du renfort relative à chaque partie est déduite essentiellement de la 
compression des pores existants dans la zone au niveau microscopique et macroscopique, ce qui induit directement une variation des paramètres porosité et perméabilité du même renfort entre les zones. En conséquences, la forme et la position du front d'écoulement ainsi que l'évolution de la pression seront réparties de manière différente entre les deux zones (figure.6).

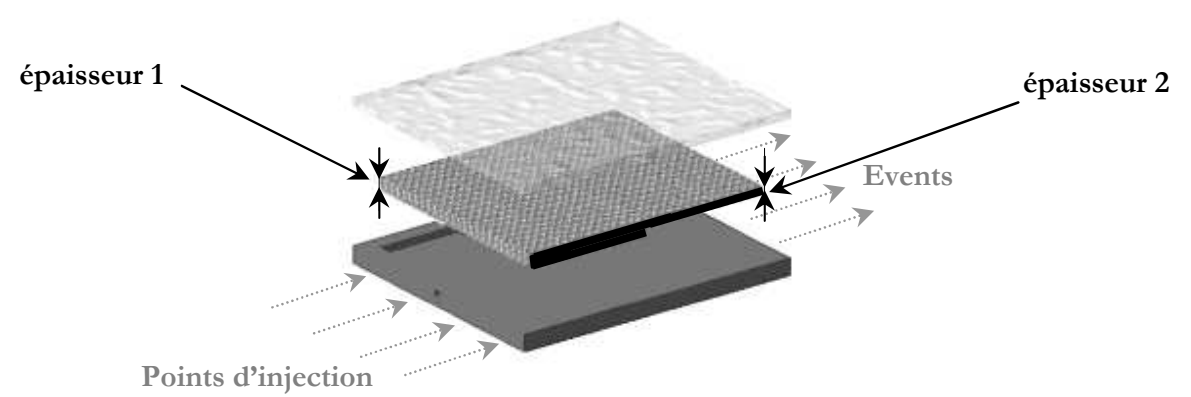

Figure 6 : Injection Unidirectionnel avec renfort à épaisseur variable
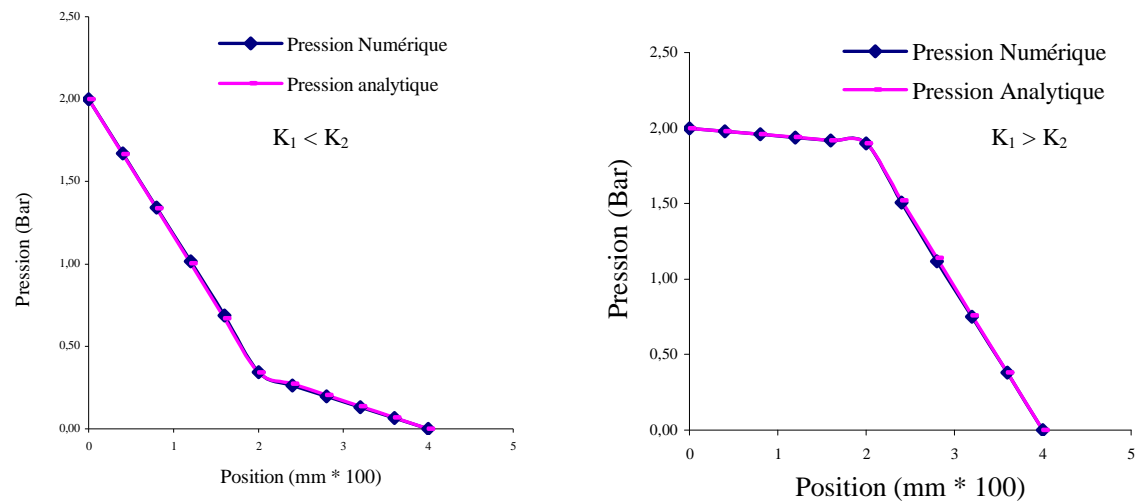

Figure 7 : Evolution de la pression en fonction de la position pour un multi épaisseur

\section{Case 2D :}

Dans le cas d'un écoulement bidimensionnel nous avons procédé à l'utilisation du même renfort à avec variation du nombre de plis, l'empreinte du moule est à épaisseur uniforme : 


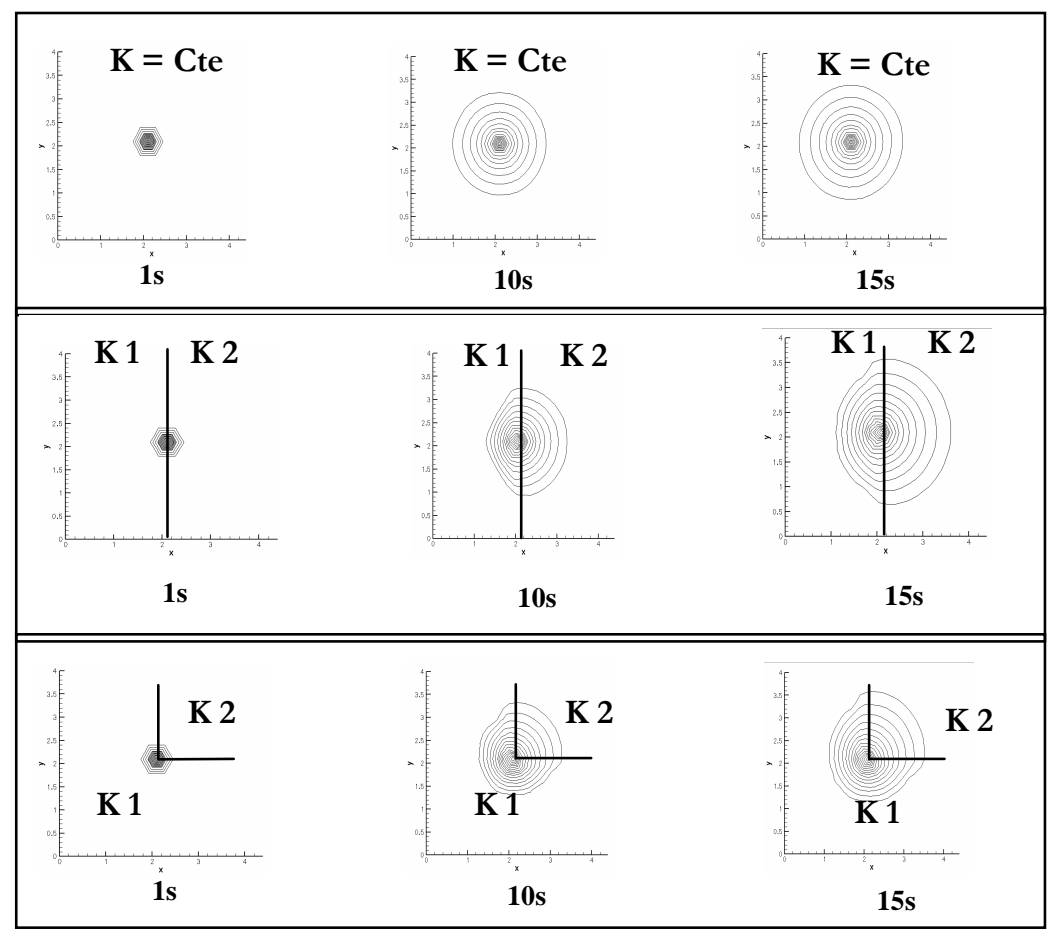

Figure 8 : Front d'écoulement pour une injection bidimensionnelle multi épaisseur

\subsection{Simulation de la distribution de la Pression pour un renfort multi-épaisseur :}

Au cours du paragraphe précédent, a été étudié l'influence du nombre de plis et des séquences d'empilement sur la forme du front d'écoulement lors du remplissage du moule en RTM. Dans ce paragraphe, seront présentés quelques exemples de résultats relatifs à l'étude de l'effet de variation de l'épaisseur du renfort sur la distribution de la pression au cours de la simulation de remplissage du moule.

La distribution de pression influence directement sur le choix de la pression d'injection, le temps de remplissage, la position des points d'injection et des évents mais surtout sur les propriétés mécaniques de la pièce finale. L'approche adoptée consiste à traiter le problème au niveau élément. L'effet de variation d'épaisseur influence directement sur la porosité et la perméabilité à l'échelle élément, l'injection de l'effet de cette variation dans le code donne une qualité bien particulière à la solution que nous proposons. 
Le modèle traité est un couvercle pour installation électrique réalisé à base d'un renfort en fibre de verre et d'une résine en polyester isophtalique. Le modèle choisi comporte la particularité de l'existence d'insert carré à l'intérieur de la pièce, avec changement d'épaisseur du renfort autour l'insert (zone carrée figure.9).

A travers cet exemple, nous allons présenter la comparaison de quelques résultats concernant la distribution de pression pour ce modèle au cours du remplissage du moule en RTM avec et sans prise en charge de l'effet de la variation de l'épaisseur

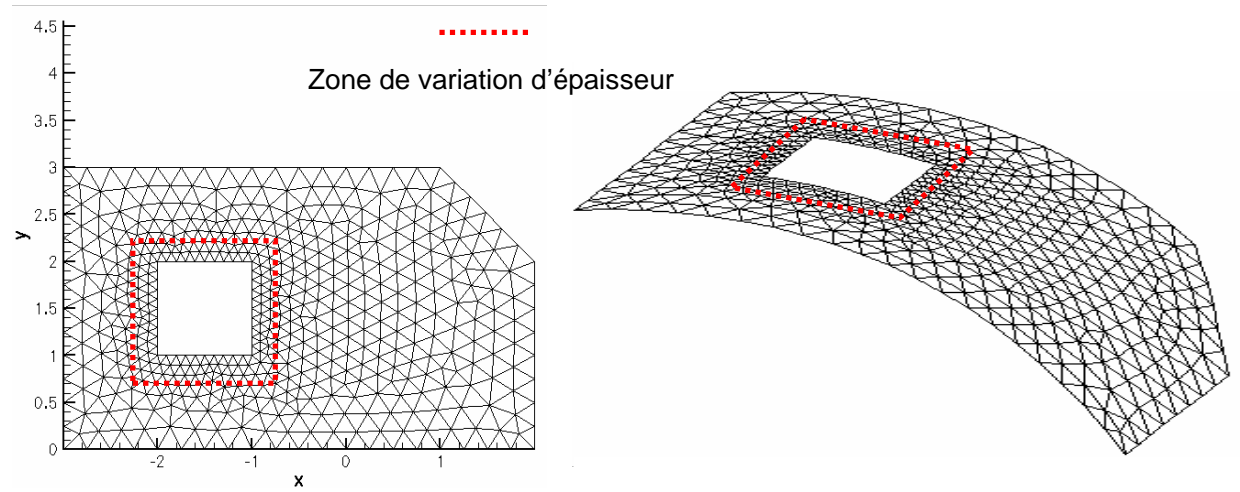

Figure 9 : Discrétisation d'une pièce avec insert et renfort à épaisseur variable

- Simulation 2D sans prise en charge de l'effet de variation d'épaisseur

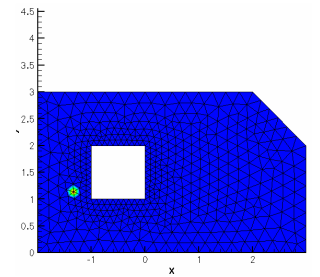

$1.05 \mathrm{sec}$

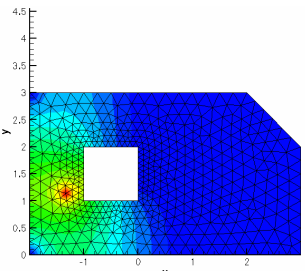

$40.67 \mathrm{sec}$

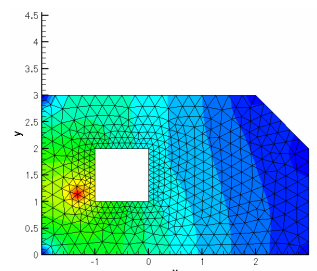

$73.81 \mathrm{sec}$ 
- Simulation 2D avec prise en charge de l'effet de variation d'épaisseur

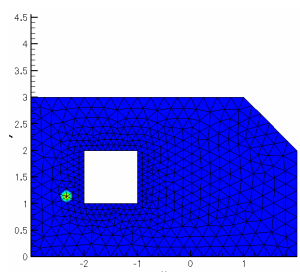

$1.05 \mathrm{sec}$

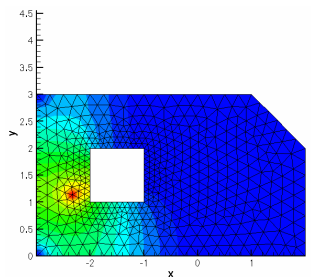

$40.67 \mathrm{sec}$

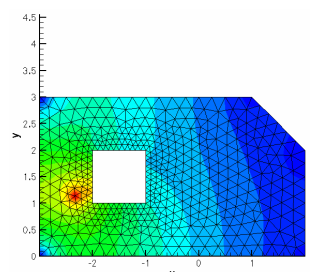

$73.81 \mathrm{sec}$

- Simulation 2.5 D de la distribution de pression avec prise en compte de l'effet de variation d'épaisseur:
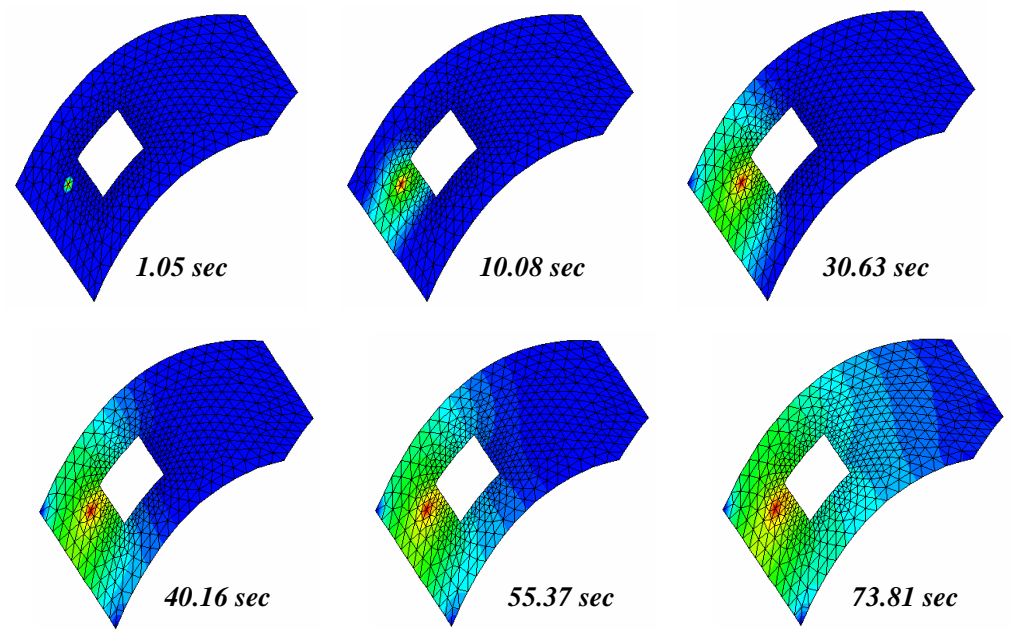

\section{Conclusions}

Dans cette étude, nous avons développé un mailleur et un code numérique pour simuler le remplissage d'un moule isotherme dans le cadre du procédé RTM en adoptant la méthode $\mathrm{CV} / \mathrm{FEM}$ et VOF, avec prise en compte de la présence d'obstacles couplée à l'effet de la variation de l'épaisseur et des couches du renfort. Les résultats de la simulation numérique montrent une parfaite concordance avec des résultats : analytiques, expérimentaux et numériques. On note l'efficacité du modèle numérique développé dans le traitement du front d'écoulement et de la distribution de pression. Une excellente reproduction de la forme du front ainsi qu'une bonne précision dans son positionnement sont obtenues. Dans nos prochaines études, nous allons nous intéresser aux effets thermiques et à l'effet capillaire. 


\section{Référence}

[1] Li, S. and Gauvin, R., "Numerical Analysis of the Resin Flow in Resin Transfer Molding," Journal of Reinforced Plastics and Composites, Vol. 10, 1991, pp. 314- 327.

[2] Trochu, F. and Gauvin, R., "Limitations of a Boundary-Fitted Finite Difference Method for the Simulation of the Resin Transfer Molding Process," Journal of Reinforced Plastics and Composites, 1992, Vol. 11, No. 7, pp. 772-786.

[3] Hattabi M., Snaike I., Echaabi J. and Bensaleh M.O., "Simulation du front d'écoulement dans les procédés de moulage des composites liquide », Comptes Rendus Mecanique, Volume 333, Issue 7, July 2005, pp. 585-591.

[4] Wang, V. W., Hieber, C.A. and Wang, K. K., "Simulation in Injection Molding of ThreeDimensional Thin Parts," In ANTEC 86, Boston, (Fairfield, Connecticut: Society of Plastics Engineer, pp. 97-102, 1986.

[5] Fracchia, C.A., Castro, J., and Tucker, Cl.L., "A Finite Element/Control Volume Simulation of Resin Transfer Mold Filling," In Proceedings of the American Society for Composites Fourth Technical Conference, Lancaster, PA, pp. 157-166, 1989.

[6] Bruschke, M.V., and Advani S.G., "A Finite Element Control Volume Approach to Mold Filling in Anisotropic Porous Media," Polymer Composites, Vol. 11, No. 6, 1990, pp. 398-405

[7] Young, W.B. al., "Analysis of Resin Injection Molding in Molds with preplaced Fiber Mats: 2.Numerical Simulation and Experiments of Mold Filling," Polymer Composites, Vol. 12, $\mathrm{N}^{\circ}$ 1, 1991, pp. 30-38

[8] Lin, R., Lee, L.J. and Liou, M., "Non-isothermal Mold Filing and Curing Simulation in Thin Cavities with Preplaced Fiber Mats," International Polymer Processing, Vol. 6, No. 4, 1991, pp. 356-369

[9] Voller, V.R., and Chen, Y.F., "Prediction of Filling Time in Porous Cavities," International Journal for Numerical Methods in Fluids, Vol. 23, No. 7. 1996, pp. 661-672

[10] Reddy, J. N., An Introduction to the Finite Element Method, McGraw Hill, Inc., New York, (1993).

[11] Moon Koo Kang, Woo Il Lee," A flow-front refinement technique for the numerical simulation of the resin-transfer molding process", Composites Science and Technology 59 (1999) pp. $1663-1674$.

[12] Lonne S. (DRT/FAR/LIST/DECS/SISC/LSUT, CEA) "Modeling of ultrasonic wave propagation in composite materials obtained by the Resin Transfer Molding Process" Thèse Année de publication 2004.

[13] B. Chen, AH-D Leng and T-W Chou, "A non linear compaction model for fibrous performs", Composites Part A, 32(5), pp. 701-707, 2001.

[14] M.J. Buntain, S. Bickerton "Compression flow permeability measurement: a continuous technique " Composites: Part A 34 (2003) 445-457 « 5 March 2003 » 